\title{
Features of Anaplastic Thyroid Carcinoma on Liquid-based Cytology and Pitfalls in the Cytologic Diagnosis of Thyroid Tumors with Spindle Cells: A Case Report
}

\author{
Songmi Noh, Eunah Shin, Jeong Yun Shim and Ji Young Kim* \\ Department of Pathology, CHA Gangnam Medical Center, CHA University, South Korea
}

Received: February 10, 2018; Published: February 20, 2018

*Corresponding author: Ji Young Kim, Department of Pathology, CHA Gangnam Medical Center, CHA University, Seoul, South Korea, 566, Nonhyon-ro, Gangnamgu, Seoul, 06135, South Korea, Tel: 82-2-3468-3058; Fax: 82-2-3468-2619; Email: jiyoung@cha.ac.kr

\begin{abstract}
Anaplastic thyroid carcinoma (ATC) is a highly aggressive but rare thyroid malignancy. Cytologically it typically shows a mixture of three cell groups, i.e. spindle cells, giant cells, and squamoid/epithelioid cells. But, when it is composed predominantly of spindle cells, the diagnosis of ATC may not be robust. Here we report a case of thyroid anaplastic carcinoma predominantly composed of spindle cells with its cytologic features on liquid-based preparation. A 61-year old male visited our hospital with a $7 \mathrm{~cm}$-sized thyroid nodule detected on a regular checkup. The initial fine needle aspiration was unsuccessful showing only cystic fluid. The patient underwent radiofrequency ablation (RFA) with decrease in the size of the mass that, after six month, enlarged back again. Left lobectomy and isthmusectomy was performed. Liquid-based cytologic sample was prepared using the scraped material from the resected mass. Cytologically, the predominant cells were spindle cells, arranged in clusters or in single cells. They had enlarged nuclei with vesicular chromatin with minimal chromatic clumping. The nucleoli were small but prominent. Giant cells, squamoid cells, or large pleomorphic cells were not present. The background was slightly necrotic and degenerative. When a thyroid FNA from a thyroid nodule reveals spindle cells, differential diagnosis should include ATC, medullary carcinoma, other sarcomas, reactive fibrosis in Riedel's thyroiditis, etc. The degree of cytologic atypia of the spindle cells, the presence of other components such as giant cells and squamoid/epithelioid cells, along with the immunohistochemical staining for calcitonin, CEA, PAX-8, and/or TTF-1 can readily differentiate ATC from others.
\end{abstract}

Keywords: Anaplastic thyroid carcinoma; Ablation therapy; Liquid-based cytology; Spindle cells

Abbreviations: ATC: Anaplastic Thyroid Carcinoma; RFA: Radio Frequency Ablation; SMA: Smooth Muscle Actin; SETTLE: Spine Cell Tumor with Thymus-Like Differentiation

\section{Introduction}

Anaplastic thyroid carcinoma (ATC) is a highly aggressive but very rare thyroid malignancy. Its cytomorphologic features, according to the previous case reports [1,2], have been described as typically showing a mixture of three cell groups, i.e. spindle cells, giant cells, and squamoid/epithelioid cells. But, when it is composed predominantly of spindle cells, without notable giant cells or epithelioid cells, the diagnosis of ATC may not be robust. Here we report a case of thyroid anaplastic carcinoma predominantly composed of spindle cells with its cytologic features on liquid-based preparation. We also discuss some pitfalls in the cytologic diagnosis of thyroid FNA with spindle cells.

\section{Case Report}

A 61-year old male visited our hospital with a thyroid nodule detected on regular check-up. Thyroid ultrasonogram showed a $7.5 \mathrm{~cm}$-sized nodule in the left lobe that extended down to the aortic arch level. There was also a $0.6 \mathrm{~cm}$-sized, partly calcified nodule in the right lobe. T3, T4, and TSH levels were within normal range. Fine needle aspiration with liquid-based cytology was performed for both nodules. The left lobe mass revealed only cystic content with few cells (2017 Bethesda system category I). The right lobe nodule was diagnosed as benign follicular lesion (2017 Bethesda system category II). The BRAF test from the aspirate of left lobe mass was 
negative. The patient underwent radiofrequency ablation (RFA) for the left lobe mass that led to the decrease in size to $2.8 \mathrm{~cm}$. But, after six months, the mass enlarged again to $4.5 \mathrm{~cm}$. Left lobectomy and isthmusectomy was performed. The left lobe was mostly replaced by a $5.5 \mathrm{~cm}$-sized, round nodule with thick fibrous capsule. The cut section was grayish white, soft to firm, solid, rubbery, and glistening with multifocal necrosis. Liquid-based cytologic sample was prepared using the scraped material from the cut surface.

Cytologically, the predominant cell population was spindle cells, arranged in clusters or in single cells. They were larger than usual fibroblasts and had enlarged nuclei with vesicular chromatin with minimal chromatic clumping. The nucleoli were small but prominent (Figure 1A). Mitoses were infrequently noted (Figure 1B). The second group of cells, only sparsely noted, was small blandlooking cells in solid aggregates or in follicular arrangements. Their nuclei were slightly hyperchromatic but not significantly atypical (Figure 1A). These cells are believed to correspond to the poorly differentiated carcinoma component of the lesion. Giant cells, squamoid cells, or large pleomorphic cells were not present. The background was relatively clear, showing some infarcted spindle cells without obvious necrotic debris. Histologically, the mass was an ATC with infarction, predominantly composed of spindle cells (Figure 1C). There also was a small fraction of poorly differentiated carcinoma with occasional follicular or insular pattern (Figure 1D). A large area of infarction with hyalinized stroma was present as well. The spindle cells were positive to cytokeratin and PAX-8, and negative to CEA, calcitonin, and p53. The patient was referred to another hospital after the operation. In 1 year follow up, he developed local recurrence and multiple lung and bone metastases and was still in the course of combined chemoradiation therapy.

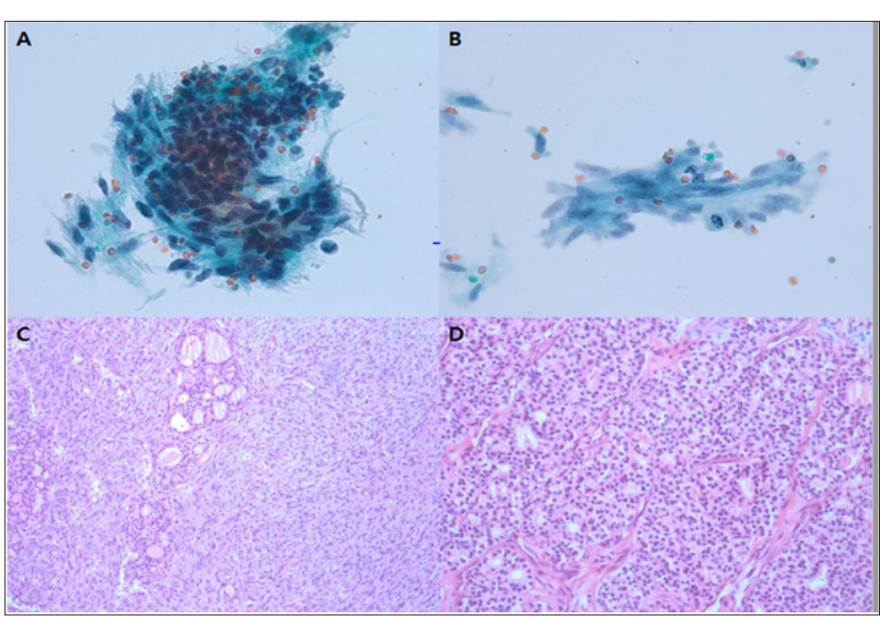

Figure 1: Pathological findings.

A. Clusters of spindle cells intermingled with round cells. $(x 400)$

B. Spindle cells with high N/C ratio and mitotic figure. $(x 400)$

C. Tumor composed of spindle cells merge with the neoplastic follicles.(x100)

D. tumor with insular pattern. (x200)

\section{Discussion}

When a thyroid fine needle aspirate reveals spindle cells, the differential diagnosis should include both malignant and benign thyroid lesions that can show spindle cells, such as ATC, medullary carcinoma, high grade sarcomas, other rare thyroid tumors with spindle cells, reactive fibrosis in Riedel's thyroiditis, etc.

Spindle cells in ATC are usually high grade showing markedly pleomorphic, hyperchromatic and enlarged nuclei with irregular nuclear membranes and occasional mitoses. In ATCs, the spindle cells are usually mixed with polymorphous epithelioid/squamoid cells and giant cells, the two other cellular groups, which altogether comprise the typical cytological feature of ATC [1]. Occasionally the giant cells dominate other cellular components in the aspirate, rendering the osteoclastoma-like features [2,3]. Anaplastic plump epithelioid/squamoid cells in ATC can sometimes look like squamous cell carcinoma, which are usually metastatic from other head and neck sites. Stromal desmoplasia, not uncommonly associated with squamous cell carcinoma, can make the differential diagnosis more difficult. The spindle cells from the desmoplastic stroma of squamous cell carcinoma lack the cytologic atypia of ATC. Immunohistochemical stain for PAX-8 is reported positive in $100 \%$ of squamoid variant and $50 \%$ in spindle variant of ATC, demonstrating the thyroid origin of the tumor [4]. Some ATCs mixed with other thyroid carcinomas such as poorly differentiated carcinoma or papillary thyroid carcinoma may show corresponding cellular components on cytology, as is in our case.

Spindle cells in medullary carcinoma are usually smaller and blander-looking with finely clumped or "salt and pepper" chromatin. Nucleoli are inconspicuous. More commonly the cells of medullary carcinoma are plasmacytoid than spindle, and scattered singly rather than in aggregates or bundles. They often accompany pinkish amorphous amyloid material in the background. Immunohistochemical staining to calcitonin and CEA can readily differentiate medullary carcinoma from ATC $[5,6]$. 
High grade sarcomas in thyroid, either primary or metastatic, are rare. Leiomyosarcomas, metastasized from uterus or more rarely primary in thyroid, appear as bundles of spindle cells on fine needle aspiration. The cytological details of nuclear enlargement and hyperchromasia with coarse chromatin are similar to those of spindle cells of ATC. The cytoplasm in leiomyosarcoma cells tends to be more fibrillar, but the difference is not distinct. Immunohistochemically, the spindle cells of leiomysarcoma are desmin and smooth muscle actin (SMA) positive and PAX8 negative, while the spindle cells of ATC are generally PAX8 positive and desmin negative with variable SMA positivity. When it comes to primary sarcomas in thyroid, spindle cells are not the major feature in FNA of angiosarcoma, the most common primary sarcoma in thyroid. It generally shows isolated large "epithelioid" cells with eosinophilic to vacuolated cytoplasm and central round nuclei containing prominent nucleoli. Difference of cytologic features of tumor cells and positivity to PAX-8 can help diagnosing ATC from angiosarcoma.

Spine cell tumor with thymus-like differentiation (SETTLE) is a very rare thyroid malignancy characterized by biphasic appearance of spindle cells blending into glandular cells [7]. They are arranged into lobules separated by sclerotic fibrous connective tissue septa. Spindle cells of SETTLE in aspirate are uniform and small with inconspicuous nucleoli. Columnar cells can be seen as well in the background along with some eosinophilic extracellular mucinous material. Both the spindle and glandular cells strongly express pancytokeratin and vimentin which are negative in ATC. Marked nuclear pleomorphism or extensive necrosis is not a common picture in SETTLE [7]. As in our case, thyroid aspiration cytology after RFA is difficult to differentiate from SETTLE because tumor necrosis is not well observed due to iatrogenic infarction and spindle cells were less pleomorphic. Riedel's thyroiditis is a rare fibrosing disorder characterized by progressive fibrosis of the thyroid. It may show bland looking spindle cells on FNA. Those spindle cells, allegedly fibroblasts, are admixed with more plump myofibroblastic spindle cells, and a small number of mainly lymphocytic inflammatory cells [8]. But, more often, it typically appear as acellular and paucicellular aspirate, thus is quite frequently non-diagnostic [7]. Differential diagnosis of ATC from Riedels's thyroiditis can be reached based primarily on the cytologic atypia and pleomorphism.
In our case, spindle cells of the tumor were admixed with bland looking follicular cells. Furthermore, tumor necrosis, a feature of ATC, was not observed because of previous ablation therapy. This is why ATC was difficult to diagnose in thyroid aspiration cytology. When a thyroid FNA reveals spindle cells, the degree of cytologic atypia of the spindle cells, the presence of other components such as giant cells, squamoid/epithelioid cells and necrotic cells, along with the immunohistochemical staining for calcitonin, CEA, PAX-8, and/or TTF-1 can readily differentiate ATC from others.

\section{Acknowledgement}

This research was supported by Basic Science Research Program through the National Research Foundation of Korea (NRF) funded by the Ministry of Science, ICT \& Future Planning (2017R1C1B5018206).

\section{References}

1. Guarda LA (1991) Anaplastic thyroid carcinoma: cytomorphology and clinical implications of fine-needle aspiration. Diagn Cytopathol 7(1): 63-67.

2. Kumar PV, S Torabinejad, GH Omrani (1997) Osteoclastomalike anaplastic carcinoma of the thyroid gland diagnosed by fine needle aspiration cytology. Report of two cases. Acta Cytol 41(4 Suppl): 13451348.

3. Mehdi G, HA Ansari, SA Siddiqui (2007) Cytology of anaplastic giant cell carcinoma of the thyroid with osteoclast-like giant cells-a case report. Diagn Cytopathol 35(2): 111-112.

4. Bishop JA, R Sharma, WH Westra (2011) PAX8 immunostaining of anaplastic thyroid carcinoma: a reliable means of discerning thyroid origin for undifferentiated tumors of the head and neck. Hum Pathol 42(12): 1873-1877.

5. Bellevicine C (2013) PAX8 is expressed in anaplastic thyroid carcinoma diagnosed by fine-needle aspiration: a study of three cases with histological correlates. Eur J Endocrinol 169(3): 307-311.

6. Talbot I, PE Wakely (2015) Undifferentiated (anaplastic) thyroid carcinoma: Practical immunohistochemistry and cytologic look-alikes. Semin Diagn Pathol 32(4): 305-310.

7. Nikiforov EB, W Thompso L (2012) Diagnostic Pathology and Molecular Genetics of the Thyroid. ( $2^{\text {nd }}$ Edn.). Wolter Kluwer/Lippincott Williams \& Wilkins, USA.

8. Weidner AS (2015) Riedel thyroiditis: Fine needle aspiration findings of a rare entity. Diagn Cytopathol 43(9): 747-750.
This work is licensed under Creative Commons Attribution 4.0 License

Submission Link: http://biomedres.us/submit-manuscript.php

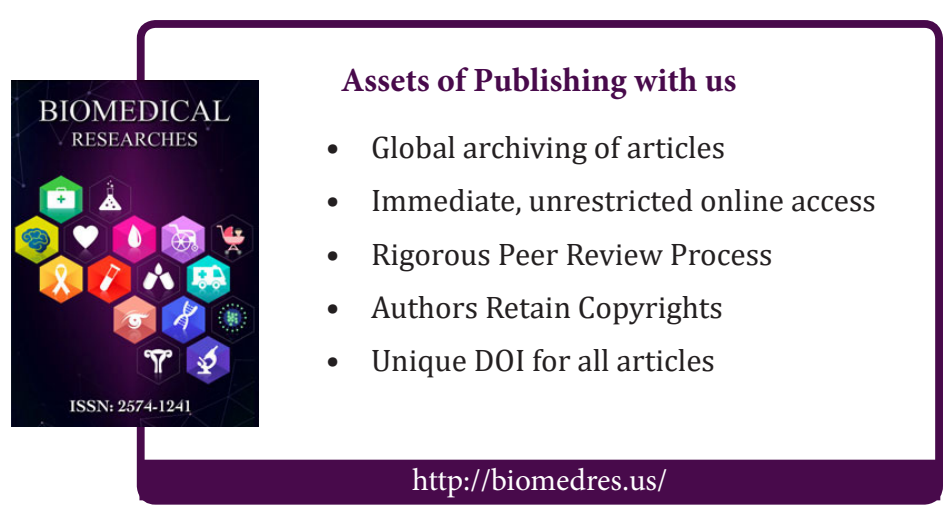

